\title{
Section 5(4)
}

\author{
Clare Bowler, Lecturer in the Psychiatry of Old Age; and Sally-AnN COOPER, Senior \\ Registrar in General Psychiatry, Mental Illness Unit, Leicester General Hospital, \\ Gwendolen Road, Leicester LE5 4PW
}

The 1983 Mental Health Act brought several changes aimed at improving the rights of detained patients. Included was a new provision safeguarding the position of nursing staff-Section 5(4); no provision equivalent to the nurses' holding power existed in the 1959 Act. Nurses previously were expected to act in good faith to restrain patients where necessary while the doctor was sought. Such actions under common law were viewed by some to provide nurses with insufficient protection. The 1983 Act made clear the legal position of nurses. A suitably qualified psychiatric nurse can compulsorily detain a hospital patient receiving treatment for a mental disorder for up to six hours. This allows time for the responsible medical officer or nominated deputy to be obtained. The mental disorder must be of a degree where immediate detention is required to protect others from the patient, or to protect the patient's own health or safety.

Despite recent interest and reports of the use of Section 5(2) of the Act (Joyce et al, 1991; Brown, 1991; Pourgourides et al, 1992; Cooper \& Harper, 1992), there is little direct reference to Section 5(4). In a recent national survey, Cooper \& Harper (1992) described how nurses in some districts are reluctant to use Section 5(4), feeling uncomfortable with the power, and preferring still to use powers of persuasion and common law for detention until the arrival of the doctor. This study reviews the use of Section $5(4)$ in a major Leicestershire psychiatric unit.

\section{The study}

An audit was undertaken of all Section 5(4)s implemented at the Leicester General Hospital from the introduction of the Act in 1983 to March 1992. The appropriate medical and nursing case-notes for each identified patient were reviewed, and information recorded about characteristics of the patients and their psychiatric illness.

\section{Findings}

Leicester General Hospital has a 150 bedded psychiatric unit which provides adult psychiatric services for a catchment area of 374,000 . This represents a city patch of 154,000 and a county patch of 220,000 .
From 1983 to March 1992, Section 5(4) had been implemented in the hospital on 99 occasions. Five patients had been detained under Section 5(4) on more than one occasion. Of the people so detained, the sex distribution was 52 women (58\%) and 37 men (42\%); mean age at implementation was 33 years (range 17-76 years). Regarding marital status, $48 \%$ of the patients were single, $40 \%$ married, $6 \%$ divorced, $3 \%$ separated, and $2 \%$ were widowed.

Of the occasions when Section 5(4) was implemented in 94 cases the person had been admitted to the hospital with informal status. In four cases, admission had been under Section 2 of the Mental Health Act, and on one occasion, admission had been under Section 4 of the Act.

For each episode of detention under Section 5(4), the ICD-9 diagnoses of the patients were determined. Manic depressive psychosis was present in 55 patients (30 patients were depressed, 19 manic and six had mixed affective states); 30 patients had schizophrenia; four patients presented with paranoid states; five patients with personality disorder; and one patient each with alcohol dependency, adjustment reaction, and anxiety state. Diagnosis was unclear in two cases. The reasons given for implementation of the Section 5(4)s were refusal to stay in hospital informally, combined with severe psychosis in 36 cases, suicide risk in $\mathbf{5 0}$ cases and risk of violence to others in 11 cases. In two cases, the reasons for implementation were not documented in the notes. For 38 of the episodes of detention, the person had a past history of deliberate self harm, and for 15 of the episodes, the person had a past history of violence to others. In some cases, instructions had been written in the medical notes indicating that they should not be allowed to self discharge.

Twenty-eight of the Section 5(4)s had been implemented on the day of admission and 49 within three days of admission. Therefore, in 50 cases the patient had been in hospital for more than three days when detained. Detention under Section 5(4) occurred on a first hospital admission in 20 cases; hence on 79 occasions the person was detained on a subsequent admission. Thirty-seven of the Section 5(4)s were completed during normal working hours, whereas 54 occurred outside of office hours. This information was unavailable in eight cases. 
Outcome was analysed in terms of application of another section of the Act, or reversal to informal status. On 78 occasions, Section 5(2) was applied to allow for detention of the patient while fuller assessment for application under the Act proceeded. In six cases, Section 2 was completed directly from Section 5(4); in one case, Section 3 was directly completed. On 14 occasions, reversion to informal status from Section 5(4) occurred; in the majority of these cases the patient had subsequently agreed to remain in hospital voluntarily.

\section{Comments}

The frequency with which Section 5(4) is implemented at this hospital reflects local psychiatric practice in Leicestershire, where appropriate medical personnel are close at hand to assess the psychiatric emergency. One would expect more frequent use of Section $5(4)$ in hospitals where the responsible medical officer has commitments to practise in the community and the nominated deputy is a nonresident doctor. In Leicester, implementation of the nurses' holding power occurred when immediate action was necessary; the patient attempting to leave, being at risk, and not amenable to persuasion. The use of Section 5(4) follows nursing policy, which at Leicester General Hospital follows the guidelines set out in the Code of Practice (1990).

The study revealed a larger number of people detained under nurses' holding power to be women, single, with a past history of deliberate self harm or violence towards others, and having a previous psychiatric admission. Some of these characteristics may represent those of the total population of psychiatric hospital admissions. Eighty-four per cent of detentions were for people with schizophrenia or manic depressive psychosis, and both of these conditions are associated with a high suicide incidence. The conversion rate of the Section 5(4)s to other sections of the Act is high at $85 \%$, suggesting that in Leicester the nurses' holding power is being used appropriately. The psychiatrists are largely in agreement with nurses' assessment of risk and support their decision to detain the patient. For the majority of cases who became informal after Section 5(4), the patient had subsequently agreed to stay in hospital voluntarily. In 28 cases, the section was enforced on the day of the patient's admission. This finding echoes that of reported studies of the use of Section 5(2). Ideally, these patients would have been admitted to hospital under Section 2 or 3 of the Act. However, the requirement for this is often not clear until shortly after the patient is in hospital.

In emergency situations, implementation of Section 5(4) by the nurse may be more appropriate than common law detention, as the Section gives a legal basis for restricting a patient's liberty. The nurse is accountable and must formally justify detention of the patient, with the circumstances being amenable to review by senior doctors, nurses and management. However, the person detained under Section 5(4) has no legal right to appeal against this decision. For this reason, audit of the use of Section 5(4) assumes great importance.

Throughout each patient's hospital admission, it is important that professionals responsible for treating that patient discuss the potential risks, should self discharge become desired. There are difficulties in projecting in time the mental state of the patient, as changes can occur hour by hour in an acutely ill patient. Attempts have been made to leave instructions about this eventuality in the medical notes of some patients. However, assessment at the time of the emergency by appropriately qualified staff is paramount. Hence it is important for the responsible nurse to receive adequate training in assessment for suicide risk and dangerousness. This training, in conjunction with instruction in the provision and use of Section 5(4) should receive a high profile for student nurses training to become registered mental nurses, and should also be provided to qualified nurses as an in-service training programme.

The emphasis of the importance of understanding the issues regarding Section 5(4) will inevitably have an effect on the frequency of use of the Section, as will attitudes of the nursing staff to their legal power. An additional factor in frequency of implementation will be staffing levels - both in terms of quantity and seniority of staff. Given sufficient time and support, a distressed psychotic patient might be persuaded by a skilled nurse to stay in hospital. However, these factors remain difficult to evaluate.

The time limit of six hours in the English Act may allow greater flexibility compared with the four hours permitted in the Scottish Act. Although a doctor is always responsible on site at the Leicester General Hospital, it may be preferable for a psychiatrist with prior knowledge of the patient to review the Section. This occasionally necessitates the longer time period if the doctor is away from the hospital. As is good clinical practice, doctors in Leicestershire endeavour to review patients with minimal delay.

\section{Acknowledgements}

We wish to thank the staff of the Medical Records Department of the Mental Illness Unit at Leicester General Hospital.

\section{References}

Brown, N. S. (1991) Section 5(2) Audit (correspondence). Psychiatric Bulletin, 15, 706. 
Code of Practice: Mental Health Act 1983 (1990). London: HMSO.

COOPER, S.-A. \& HARPER, R. (1992) Section 5(2): Who acts as the consultant's nominated deputy? Psychiatric Bulletin, 16, 759-761.
Joyce, J. P., Morris, M. B. \& Palia, S. S. (1991) Section 5(2) Audit. Psychiatric Bulletin, 15, 224-225.

Pourgourides, C., Prasher, V. P. \& Oyebode, F. (1992) Use of Section $5(2)$ in clinical practice. Psychiatric Bulletin, 16, 14-16.

\title{
Too high a hurdle? The use of pre-assessment questionnaires in psychotherapy
}

\author{
TERri Eynon, Registrar in Psychotherapy; and StePhen Gladwell, Consultant \\ Psychotherapist, Uffculme Clinic, Moseley, Birmingham B13 8QD
}

Up to one third of patients referred for psychotherapy fail to attend for their first appointment (O'Loughlin, 1990). Psychotherapy assessments are usually allocated a considerable portion of uninterrupted time, and an unexpected non-attendance wastes significant clinical resources. A variety of strategies have been used to ensure that assessors are not left waiting for a patient who never comes. One method is to send out forms which must be completed and returned before a first appointment date is given. In O'Loughlin's study, in which a similar questionnaire to that detailed in this paper was used, it was suggested that sending a preappointment questionnaire reduced the default rate.

\section{The study}

This audit study arose out of a discussion among the staff in a clinic where one team (team A) used a mandatory pre-assessment questionnaire and another (team B) did not. The use of a questionnaire can delay sending out the appointment if patients are slow in returning them and also put extra demands on secretarial staff. With new standards in patient care, any administrative matter which may increase waiting times needs to be clinically justified. This led to a need to re-evaluate the use of questionnaires, and to observe their effect on the numbers of patients failing to attend.

The Uffculme Clinic, the West Midlands Regional Centre for Psychotherapy, takes referrals from primary care and from psychiatric clinics. Personal referrals are usually seen by the named consultant or one of their team, with the larger number of general clinic referrals being allocated to the teams according to their workload.

In team $A$, on receiving the referral, the patient is sent a questionnaire and asked to return it completed in 21 days. The patient is then allocated to one of the team members, and an appointment sent out. The patient is asked to confirm that they will attend by returning an acceptance card within 14 days. In team $B$, referred patients are sent an appointment and asked to confirm as above within 21 days.

The questionnaire is based upon one used at the Tavistock Clinic. The questions are designed to encourage the patient to think about their difficulties and how they relate to their earlier life and current circumstances. They may also indicate the person's motivation to work psychodynamically, and may help in the process of allocating patients to trainee assessors.

Clinic computer records and original files for all patients referred (total 695) to the clinic in 1991 were obtained, and data concerning the two teams were analysed. Failure to attend and outcome at assessment were used to determine whether or not the use of the questionnaire was beneficial in reducing the "waste" of new patient assessment time.

Patients may fail to attend their appointments in a number of ways, and this was coded as follows:

DNRQ = did not return questionnaire (team A only) ANC = appointment not confirmed $\mathrm{CBP}=$ cancelled by patient

DNA = did not attend on the day of appointment (having previously confirmed that they would attend). 